\title{
Oncology Informatics: Status Quo and Outlook
}

\author{
Paul Martin Putora ${ }^{a, b} \quad$ Michael Baudis $^{c}$ Beth M. Beadle ${ }^{d}$ Issam El Naqa ${ }^{e}$ \\ Frank A. Giordano ${ }^{f}$ Nils H. Nicolayg, h \\ ${ }^{a}$ Department of Radiation Oncology, Kantonsspital St. Gallen, St. Gallen, Switzerland; bepartment of \\ Radiation Oncology, University of Bern, Bern, Switzerland; 'Department of Molecular Life Sciences and Swiss \\ Institute of Bioinformatics, University of Zurich, Zurich, Switzerland; dDepartment of Radiation Oncology, Stanford \\ University, Stanford, CA, USA; 'Department of Radiation Oncology, University of Michigan, Ann Arbor, MI, USA; \\ fDepartment of Radiation Oncology, University of Bonn, Bonn, Germany; ${ }^{9}$ Department of Radiation Oncology, \\ University of Freiburg - Medical Center, Freiburg, Germany; ${ }^{\mathrm{h}}$ German Cancer Consortium Partner Site Freiburg, \\ German Cancer Research Center, Heidelberg, Germany
}

\section{Keywords}

Oncology · Radiation oncology · Informatics - Cancer . Artificial intelligence $\cdot$ Machine learning Electronic health record - Electronic patient-reported outcome measures

\begin{abstract}
Oncology has undergone rapid progress, with emerging developments in areas including cancer stem cells, molecularly targeted therapies, genomic analyses, and individually tailored immunotherapy. These advances have expanded the tools available in the fight against cancer. Some of these have seen broad media coverage resulting in justified public attention. However, these achievements have only been possible due to rapid developments in the expanding field of biomedical informatics and information technology (IT). Artificial intelligence, radiomics, electronic health records, and electronic patient-reported outcome measures (ePROMS) are only a few of the developments enabling further progress in oncology. The promising impact of IT in oncology will only become reality through a multidisciplinary approach to the complex challenges ahead.
\end{abstract}

(c) 2020 S. Karger AG, Basel
Oncology has undergone rapid progress, with emerging developments in areas including cancer stem cells, molecularly targeted therapies, genomic analyses for diagnostic and therapeutic stratification, and, most recently, individually tailored immunotherapy. These advances have expanded the tools available in the fight against cancer. Some of these have seen broad media coverage, often in line with the hype cycle model [1], and resulting in justified public attention. However, these achievements have only been possible due to rapid developments in the expanding fields of biomedical informatics and information technology (IT), a fact that often escapes public recognition. Exemplary advances in informatics methodologies and tools have been made in surgical and radiation oncology, in image processing for radiologic diagnostics and treatment, in histopathological classification as well as data analysis for follow-up assessment. These developments have not only resulted in increased efficiency of oncological procedures, but also in favourable survival rates, reduced treatment toxicity, and improved quality of life for many cancer patients $[2,3]$.

In modern clinical practice, tight integration of IT into workflows enables the efficient processing of rapidly ac-

\footnotetext{
Paul Martin Putora

Department of Radiation Oncology

Kantonsspital St. Gallen

Rorschacher Strasse 95, CH-9007 St. Gallen (Switzerland)

20oiedit@data00.com
} 
cumulating information enabling entirely new applications $[4,5]$. There is an increasing interest in data mining of clinical narratives from electronic medical records to utilise diagnoses and clinical characteristics for the datadriven identification of clinical trial eligibility and especially for the prediction of clinical outcomes [6]. Although the shift from paper to digital goes beyond availability and acquisition speed, the real improvement lies in the implementation of "unstructured-to-structured" data transformation. For data that is to be used in subsequent analyses, efficient mapping to computationally useable formats is essential [7]. While structured reporting alone may suffer from loss of "verbatim" information, it allows for an automated interpretation of meaning, enabling automated analyses otherwise not possible [8]. However, the transformation of unstructured data using natural language processing and other "intelligent" text parsing technologies can preserve the original textual context in addition to providing the structured data for analyses.

IT can help determine patterns emerging from traditional and high-throughput molecular data, improve diagnostics of new cancers, and assist stratification of existing cases. Emerging artificial intelligence methodologies, such as "deep learning", have been applied successfully, for example, to the detection of lung cancers on screening CT scans, breast cancers on mammography and skin cancers on digital photographs [9-12]. Furthermore, radiomic analyses of images have found new features that can be predictive of outcomes and may help determine care decisions [13]. While not every result is universally applicable [14], the use of machine learning technologies is on its way into decision support systems for imaging and other types of complex clinical data [14].

A better understanding of how we deal with information and make decisions can improve our decision-making process. By expanding our understanding of the clinical situation beyond laboratory and imaging values, a variety of parameters may become apparent [15], and, based on an IT-supported approach, criteria rarely implemented in decision-making can be identified [16]. Parameters not traditionally considered in stratification procedures are patient preferences and psychophysiological factors, which in the future may become better integrated into our data models [17]. So far, a major obstacle has been the lack of standardized collection procedures and notations for psychophysiological raw data to draw objective conclusions about these factors and their impact. Traditionally, data acquisition occurred in offices, outpatient clinics or trial units following a formalised schedule. With the spread of mobile technology, structured high-quality data (electronic patient-reported outcomes) can be submitted from the patients themselves and become directly integrated into their records [18]. It has been demonstrated that this is feasible in a general population [19-21], and the acceptance rates of such solutions both on the patient side and the side of healthcare providers appear high. Electronicpatient-reported outcomemeasures(ePROMS) enable structured first-hand data and open up the possibility to analyse the psychophysiological background of a reported incident as patient-centred IT may soon be able to connect such reports with measurements from miniature, wearable sensors and devices [22]. Newer classes of machine learning applications will focus on integrating human perception and self-expressed observations with diagnostic tests and high-frequency measurements of physiological parameters to predict adverse events and allow early therapeutic interventions.

The amount of data from electronic health records, diagnostic procedures and molecular screening analyses provides new opportunities for diagnostics, therapy and event prediction. The proliferation of electronically accessible, patient-related data will also require new or modified regulations and will provide additional entry points for security threats. From this, a need will arise to re-evaluate aspects of confidentiality, consent and patient privacy. Besides the implementation of "best practice" data storage and handling procedure, new computational approaches, such as blockchain technologies, may potentially provide building blocks of future secure data transactions [5].

The increasing qualities and quantities of data available for patient-centric analyses in oncology lend themselves to new types of "artificial intelligence" approaches, such as machine/deep learning algorithms that enable insights into information that may not be intuitively accessible [23]. However, one of the difficulties of the complexity resulting from these algorithms, particularly in deep learning scenarios, is that we are not able to intuitively evaluate their results. Here, visual approaches have been designed to help us understand the essence behind that flood of data $[24,25]$. There is a strong drive in the machine learning community to devise what is referred to as "interpretable or explainable methods" [26]. Even further, a sensible processing of the plethora of structured data may enable aided decision-making regarding complex oncological therapies by incorporating information such as patient preference and experience of the physician. In this respect, oncology informatics may eventually provide a personalised and individualised path for each patient through the often complex and multidisciplinary treatment algorithms. 
The promising impact of IT in oncology will only become reality through a multidisciplinary approach to the complex challenges ahead.

\section{Disclosure Statement}

The authors have no conflicts of interest to declare.

\section{Funding Sources}

I.E.N. declares funding by the National Institute of Health (NIH) from grants: R37-CA222215 and R01-CA233487.

\section{Author Contributions}

All authors contributed to the structure and writing of this review.

\section{References}

1 Fenn J, Linden A. Understanding Gartner's hype cycles. Strategic analysis report No. R-20-1971. Gartner, Inc; 2003. p. 88

2 Basch E, Deal AM, Dueck AC, Scher HI, Kris MG, Hudis C, et al. Overall survival results of a trial assessing patient-reported outcomes for symptom monitoring during routine cancer treatment. JAMA. 2017 Jul 11;318(2):1978

3 Denis F, Lethrosne C, Pourel N, Molinier O, Pointreau Y, Domont J, et al. Randomized trial comparing a web-mediated follow-up with routine surveillance in lung cancer patients. J Natl Cancer Inst. 2017 Sep 1;109(9).

4 Ahlbrandt J, Lablans M, Glocker K, StahlToyota S, Maier-Hein K, Maier-Hein L, et al. Modern information technology for cancer research: What's in IT for me? An overview of technologies and approaches. Oncology. 2018 Nov 15. https://doi.org/10.1159/000493638.

5 Dubovitskaya A, Novotny P, Xu Z, Wang F. Applications of blockchain technology for data-sharing in oncology: Results from a systematic literature review. Oncology. 2019 Dec. https://doi.org/10.1159/000504325.

6 Savova GK, Danciu I, Alamudun F, Miller T, Lin C, Bitterman DS, et al. Use of natural language processing to extract clinical cancer phenotypes from electronic medical records. Cancer Res. 2019;79(21):5463-70.

7 Henkel M, Stieltjes B. Structured data acquisition in oncology. Oncology. 2019 Nov. https://doi.org/10.1159/000504259.

8 Hewer E. The oncologist's guide to synoptic reporting: A primer. Oncology. 2019 Jun. https://doi.org/10.1159/000500884.

9 Esteva A, Kuprel B, Novoa RA, Ko J, Swetter SM, Blau HM, et al. Corrigendum: Dermatologist-level classification of skin cancer with deep neural networks. Nature. 2017; 546(7660):686-18.
10 Ardila D, Kiraly AP, Bharadwaj S, Choi B, Reicher JJ, Peng L, et al. End-to-end lung cancer screening with three-dimensional deep learning on low-dose chest computed tomography. Nat Med. 2019;25(6):954-61.

11 Shen L, Margolies LR, Rothstein JH, Fluder E, McBride R, Sieh W. Deep learning to improve breast cancer detection on screening mammography. Sci Rep. 2019;9(1):12495-12.

12 El Naqa I, Haider MA, Giger ML, Ten Haken RK. Artificial Intelligence: reshaping the practice of radiological sciences in the 21st century. Br J Radiol. 2020 Feb;93(1106): 20190855.

13 Lambin P, Leijenaar RT, Deist TM, Peerlings J, De Jong EEC, Van Timmeren J, et al. Radiomics: the bridge between medical imaging and personalized medicine. Nat Rev Clin Oncol. 2017 Dec;14(12):749-762.

14 Haibe-Kains B, Adam GA, Hosny A, Khodakarami F, Board M, Waldron L, et al. The importance of transparency and reproducibility in artificial intelligence research. arXiv preprint. arXiv:2003.00898. 2020.

15 Glatzer M, Panje C, Sirén C, Cihoric N, Putora $\mathrm{P}$. Decision making criteria in oncology. Oncology. 2018 Sep. https://doi.org/10.1159/ 000492272

16 Iseli T, Fischer G, Panje C, Glatzer M, Hundsberger $T$, Rothermundt $C$, et al. Insular decision criteria in clinical practice: Analysis of decision-making in oncology. Oncology. 2020. https://doi.org/10.1159/000508132.

17 Treffers T, Putora P. Emotions as social information in shared decision-making in oncology. Oncology. 2020 Jan. https://doi.org/ $10.1159 / 000505341$.

18 Giordano F, Welzel G, Siefert V, Jahnke L, Ganslandt T, Wenz F, et al. Digital follow-up and the perspective of patient-centered care in oncology: What's the PROblem?. Oncology. 2018 Dec. https://doi.org/10.1159/000495294.
19 El Shafie RA, Weber D, Bougatf N, Sprave T, Oetzel D, Huber PE, et al. Supportive care in radiotherapy based on a mobile app: Prospective multicenter survey. JMIR Mhealth Uhealth. 2018 Aug;6(8):e10916.

20 Kessel KA, Vogel MM, Alles A, Dobiasch S, Fischer H, Combs SE. Mobile app delivery of the EORTC QLQ-C30 questionnaire to assess health-related quality of life in oncological patients: usability study. JMIR Mhealth Uhealth. 2018 Feb;6(2):e45.

21 Pavic M, Klaas V, Theile G, Kraft J, Tröster G Guckenberger M. Feasibility and usability aspects of continuous remote monitoring of health status in palliative cancer patients using wearables. Oncology. $2019 \mathrm{Jul}$. https://doi. org/10.1159/000501433.

22 Gupta A, Stewart T, Bhulani N, Dong Y, Rahimi Z, Crane K, et al. Feasibility of wearable physical activity monitors in patients with cancer. JCO Clin Cancer Inform. 2018 Dec: $2: 1-10$.

23 Tseng H, Wei L, Cui S, Luo Y, Ten Haken R, El Naqa I. Machine learning and imaging informatics in oncology. Oncology. 2018 Nov. https://doi.org/10.1159/000493575.

24 Wortham RH, Theodorou A, Bryson JJ. Improving robot transparency: Real-time visualisation of robot AI substantially improves understanding in naive observers. In: 2017 26th IEEE International Symposium on Robot and Human Interactive Communication (RO-MAN), Lisbon, 2017. p. 1424-31.

25 Schlachter M, Preim B, Bühler K, Raidou R. Principles of visualization in radiation oncology. Oncology. 2020 Jan. https://doi. org/10.1159/000504940.

26 Luo Y, Tseng H-H, Cui S, Wei L, Ten Haken RK, El Naqa I. Balancing accuracy and interpretability of machine learning approaches for radiation treatment outcomes modeling. BJR Open. 2019;1(1):20190021. 